\title{
TheNNT.com: The Concept, The Mission, AND the History
}

\begin{abstract}
Department of Emergency Medicine, State University of New York, Downstate Medical Center. New York City, New York, United States of America.
\end{abstract} ORCID: 0000-0002-6427-986X. Shahriar.Zehtabchi@downstate.edu

In the age of information and shared decision making, both patients and physicians need accurate, unbiased, digestible information. Trustworthy, summarized data on benefits, harms, costs and other potential consequences of medical decision making should be available to all stake holders.

No medical decision is easy. For patients, virtually all decisions represent life changing burdens. Antihypertensive drugs, for instance, may be casually recommended and prescribed, but for patients the commitment is far from casual: a lifetime of daily pills, financial costs, physician visits, blood pressure checks, refills, chemical dependency, side effects, and the burden of being labeled 'ill', to name a just a few of the indignities that accompany a new prescription.

Moreover, a single chest $\mathrm{x}$-ray can lead to serious harm. Incidental or false positive findings, potentially unnecessary procedures, radiation, and invasive treatments may follow'. latrogenic fear of results, of serious illness, of loss, and of further treatment, can be constant and devastating for patients and families. Even the smallest medical decision therefore has consequences profoundly affecting a persons' well-being. The harms, burdens, and life alterations associated with medical intervention are justifiable only when these factors are trumped by scientific proof of benefit-proof patients and families assume underlies virtually every prescription, treatment, and test.

One way of communicating such information is Number-Needed-To-Treat, the NNT. NNT implicitly acknowledges not everyone is helped by an intervention-some benefit, some are harmed, and most are not measurably affected. The NNT and the NNH tell us how many will likely fall into each category. The core value of the NNT is simple, honest, complete communication of scientific data.

The 'number' in NNT derives from the percentage of patients who experience the desired outcome in an active treatment versus a control group. It gives us a numerical sense of efficacy by indicating how many people, on average, were treated with an intervention in trials for one additional person to experience the desired outcome ${ }^{2}$. For example: 5 children need to be vaccinated with influenza vaccine to prevent one case of influenza-NNT=53.

NNT is intuitive, easy to understand for both clinicians and patients, and thus an effective tool to facilitate communication and inform decisions. It is calculated simply, dividing 1 by the absolute risk 
reduction. The concept applies to harmful outcomes as well. The number needed to harm (NNH) tells us how many were treated for one additional person to be harmed by an intervention. For instance, among every 127 adults receiving the influenza vaccine one experiences fever because of the vaccine. Informed decisions consider both NNTs and $\mathrm{NNHs}^{3}$.

Where to get the NNTs: TheNNT.com ${ }^{4}$ is an open access, independent medical reference, developed by a multidisciplinary physician group with no financial ties to industry or other funding agencies. The website is not limited to any specialty and publishes appraised evidence-based summaries on a broad variety of topics. Content is peer-reviewed by internal and external reviewers and updated when new research emerges.

The History of TheNNT.com: More than a decade ago a small group of dedicated physicians met to consider how to create an open access, unbiased storehouse of evidence designed to convey the best available data on medical interventions. Among them was Graham Walker, one of the founders of MDCalc, the popular clinical decision rule medical reference ${ }^{5}$. Dr. Walker took the NNT concept and ran with it, developing simple graphics and intuitive interfaces to display NNT content, while the rest of the group began producing and editing content, with the intention of becoming an online peer reviewed resource for evidence based summaries. Today, TheNNT.com exists under the umbrella of MD Aware, the parent company of MDCalc, and continues to expand its reach as the most trusted name in critically appraised synopses. Besides millions of page views and dozens of new peer reviewed summaries each year, TheNNT continues to increase its publishing collaborations with scientific journals such as Academic Emergency Medicine journal, American Family Physician journal, and the Journal of Evidence-based Healthcare.

The visual display: In addition to numerical presentations TheNNT.com employs a color recommendation to help summarize evidence using judgment-based assessments of harm-benefit balance. Green suggests patient-important benefits that trump harms; Yellow suggests data is not conclusive or substantial enough for a clear rating; Red suggests benefits do not outweigh harms (and thus, based on unmeasured costs and burdens we consider the impact of the intervention to be negative overall); and Black suggests harms clearly trump benefits. Color recommendations are decided by consensus among authors, peer-reviewers, and editorial board members ${ }^{4}$.

Strength of NNT: The NNT is a simple way to communicate treatment and harm effects ("roughly 1 in 70 adults is likely to avoid influenza because of the vaccine, while about 1 in 100 or less will also experience fever"). Other indices such as $p$ values, relative risks or odds ratios are non-intuitive, even for most clinicians. They are hard to conceptualize and hard to explain. Using NNT and NNH can facilitate and promote shared decision making and helps prevent over-estimating benefits or underestimating harms, by expressly including those who see no measurable effect, i.e. the great majority, in most situations. This group (those unaffected by treatment) has traditionally been left out of most communication, leading to profound misperceptions about harms and benefits².

NNT considerations: As with any attempts to summarize or simplify, with the NNT some information is missing. For example, how often an outcome occurs at baseline cannot be extracted from the NNT. If the rate of a desirable outcome is $90 \%$ in the control group and $95 \%$ in the study group, the NNT is 20 . And if event rates are $5 \%$ and $10 \%$, respectively, the NNT is also 20 . Patients may want to know when there is a $95 \%$ probability of a desired outcome even without treatment, or a $90 \%$ chance of an undesirable outcome regardless of treatment.

Another important issue to be considered when NNT is interpreted is the risk level in the target population: a high-risk group will likely have a lower NNT (a smaller number of patients would need to be treated to observe one favorable outcome) than a low risk group. Therefore, it is important for the clinician to estimate if the patient they are treating is properly represented by the group that was studied.

The NNT also does not reflect the importance of an outcome. For example, after out-of-hospital cardiac arrest, making it to the hospital alive has a different value than complete neurological recovery. The NNT is only as valuable as the outcome it represents, thus selecting that outcome is of utmost importance ${ }^{2}$. Therefore, when comparing the benefits (NNT) and harms (NNH), it is important to consider what the outcome measured is for both. 
NNT can also only be applied to binary outcomes (yes or no, dead or alive, hospitalized or not hospitalized) and cannot be calculated for continuous or ordinal variables such as length of stay, or pain level ${ }^{2}$.

Lastly, the NNT, because it depends on outcomes used in research trials, does not integrate financial costs and the many other burdens that accompany any treatment. Physicians should keep this in mind when discussing treatment effects with patients.

Like Cates' plots, natural frequencies, and other communication devices the NNT should be wielded carefully, and with close attention to whatever works best. Individuals perceive and integrate information differently, and no single device will ever work best for everyone. Ultimately, using TheNNT.com website can, we sincerely hope, move people closer to the truth about healthcare interventions, and closer to each other in a longer journey towards leveling the playing field by sharing information.

\section{Competing interests}

Dr. Zehtabchi reports that he is the editor-in-chief for the the website discussed in the manuscript (https://www.TheNNT.com).
6. Wiley Online Library. Academic Emergency Medicine. [Internet]. 2019. [accessed 2019 Sep. 30]. Available at: https://www. onlinelibrary.wiley.com/journal/15532712

7. American Family Physician. Toilet Training. [Internet]. 2019. [accessed 2019 Sep. 30]. Available at: https://www.aafp.org/ journals/afp.html

8. Journals BAHIANA. Journal of Evidence-Based Healthcare. [Internet]. 2019. [accessed 2019 Sep. 30]. Available at: https:// www5.bahiana.edu.br/index.php/evidence

\section{References}

1. Prasad V, Lenzer J, Newman DH. Why cancer screening has never been shown to "save lives"--and what we can do about it. BMJ. 2016;352:h6080. doi: 10.1136/bmj.h6080

2. Saver JL, Lewis RJ. Number Needed to Treat: Conveying the Likelihood of a Therapeutic Effect. JAMA. 2019;321(8):798-799. doi: 10.1001/jama.2018.21971

3. The NNT. Vaccines for Preventing Influenza in Healthy Individuals. [Internet]. 2018. [accessed 2019 Sep. 30]. Available at: https://www.thennt.com/nnt/vaccines-preventing-influenzahealthy-individuals

4. The NNT. Quick summaries of evidence-based medicine. [Internet]. 2018.[accessed 2019 Sep. 30]. Available at:https://www. thennt.com

5. Mdcalc. [Internet]. 2019. [accessed 2019 Sep. 30]. Available at: https://www.mdcalc.com 